\title{
Novel Coupling of a Capacitive Probe with a Dynamic Vapor Sorption (DVS) Instrument for the Electrostatic Measurements of Powders
}

Karolina Biegaj ${ }^{1}$, Jin W. Kwek ${ }^{2}$, Tim Lukas ${ }^{3}$, Martin Rowland ${ }^{3}$, Jerry Y. Y. Heng ${ }^{1, *}$

${ }^{1}$ Department of Chemical Engineering, Imperial College London, South Kensington Campus, London, SW7 2AZ, United Kingdom.

${ }^{2}$ Institute of Chemical and Engineering Sciences, 1 Pesek Road, Jurong Island, Singapore 627833.

${ }^{3}$ Discovery Park, Pfizer Pharmaceutical Sciences, Sandwich, United Kingdom.

\footnotetext{
${ }^{*}$ Corresponding author.
}

Tel.: +44 (0)20 7594 0784; E-mail: jerry.heng@imperial.ac.uk (Jerry Y. Y. Heng). 


\begin{abstract}
Powders may become electrostatically charged due to friction. This phenomenon may lead to unusual outcomes such as increased cohesion, adhesion and poor flow. It is still a challenge to investigate the electrostatic properties of powders due to restrictions associated with existing methods. The objective is to present a novel coupling method with the Dynamic Vapor Sorption (DVS) instrument using a capacitive probe to measure the electrostatic potential of powders. The critical factors affecting measurements are defined to aid experimental design. The method was tested with glass beads tribocharged after contact with stainless steel to assess the feasibility of the setup via analysis of the repeatability and reproducibility of the data generated. The results obtained show that electrostatic measurements can be performed with minimum noise interference, short equilibration time of $15 \mathrm{~min}$ and high reproducibility based on 19 measurements. Stability of experimental conditions of $\pm 0.1{ }^{\circ} \mathrm{C}$ and $\pm 0.2 \% \mathrm{RH}$ is achieved.
\end{abstract}

Keywords:

Electrostatic potential; powder; capacitive probe; triboelectric charge; glass beads; DVS 


\section{Introduction}

Electrostatic phenomena are an integral part of any process involving the handling of loose powders, such as grinding, sieving or conveying. ${ }^{1}$ The ability of powders to become electrostatically charged has found many applications, for example in the coating industry or electrophotography, but uncontrolled charge build-up may lead to operational problems such as equipment blockage or even hazardous dust explosions. ${ }^{2}$ These events are particularly relevant to food and pharmaceutical powders, which tend to exhibit insulating properties that prevent effective electrostatic discharge..$^{3-4}$

Over the past few years, a progressive awareness and interest in electrostatic properties of powders has been observed, yet the overall knowledge still remains very limited. ${ }^{2}$ The difficulties encountered in studies on charge accumulation mainly arise from a large number of factors influencing the process and measurements. These include powder physical properties such as particle size ${ }^{5-8}$ and shape, surface roughness or crystallinity, ${ }^{9}$ environmental conditions namely humidity and temperature, ${ }^{10-11}$ as well as type of the surface that powder is in contact with. ${ }^{10}$ In addition, factors controlling the interaction between the particles and contact surface itself, such as duration of contact, might affect the charge observed. ${ }^{12}$ Consequently, quantification and hence prediction of electrostatic properties of powders can prove to be challenging, and sometimes tend to be neglected. ${ }^{13}$

From the experimental point of view, no standard procedure or method exist for quantification of charge levels acquired by powders. ${ }^{6,14}$ Even though several instruments can be used to measure the electrostatic charge of materials, the Faraday Cage, and its modified versions, remain the 
most often applied technique albeit having its own limitations. ${ }^{14}$ These include uncertainties in measurements due to a variety of reasons ranging from particle-to-wall adhesion during sampling to cleaning procedures adopted. ${ }^{6}$ The overall complexity of the tribocharging process often results in inconsistent execution of experiments. This can lead to significant data variability and hence little correlation between observed trends and assumed models. ${ }^{12-13,}{ }^{15-17}$ Instances of an alternative method, based on the Kelvin probe principle, have been fairly successful in measuring the electrostatic behaviours of various materials. ${ }^{17-19}$ This method has recently become more popular due to its non-intrusive mode of operation. ${ }^{20}$

In the present study, the potential of the capacitive probe is further extended by coupling with a commercial dynamic (gravimetric) vapour sorption (DVS) system in a simple setup. The main objective is to develop an accurate and reliable system and to explore its ability for conducting electrostatic measurements of tribocharged powders. Therefore, the aim is to be able to use the method developed in precisely controlled environmental conditions, provide good shielding from any external noise and minimize number of experimental steps that could result in additional charge transfer. Several important factors affecting the measurements were identified and evaluated during the setup development stage. These will be discussed in the paper to provide a better insight into experimental design for electrostatic measurements. The coupled setup will be useful for industries that face powder adhesion problems arising from tribocharging by simulating various environmental conditions whilst simultaneously measuring the electrostatic charge gained upon in-situ contact friction with different surfaces. 


\section{Materials and methods}

A detailed description of the operation of the capacitive probe connected to an electrostatic voltmeter is given by Pritchard et al. ${ }^{21}$ Briefly, once a measuring probe is placed close to a charged surface, a displacement current is generated as a result of probe vibrations in the electrostatic field. The converted and amplified signal is fed back to the electrode as a voltage, matching the electrostatic potential on the charged test surface measured. Overall, the system operates under the 'null method', as the measured potential occurs when no current is present between the charged surface and sensing electrode. ${ }^{21-22}$

The electrostatic probe and surface under the test can be considered as a parallel plate capacitor having plate area A separated by distance $\mathrm{D}_{0}$ (Eq. (1)). ${ }^{22}$

$Q=U \cdot C=U \cdot \frac{\epsilon \varepsilon_{0} A}{D_{0}+D_{1} \sin (\omega t)}$

The voltage between the plates is given by $\mathrm{U}, \mathrm{C}$ denotes the capacitance, whereas $\varepsilon$ and $\varepsilon_{0}$ are relative electric permittivity of the material between the plates and the electric permittivity of vacuum respectively. The second term in the denominator accounts for the probe vibrations over time $t$, hence $D_{1}$ is the amplitude of vibrations of the probe and $\omega$ is the circular frequency of vibration. For a given charge $\mathrm{Q}$, the potential experienced by the sensor depends on the separation of plates $\mathrm{D}_{0}$, therefore the positioning of the probe is important for measurement consistency. 


\subsection{Experimental setup design}

A high-resolution round electrode, having an end-view configuration sensor, (model 6000B-5C, Trek Inc., USA) is connected to an electrostatic voltmeter (Model 347, Trek Inc., USA). The vibrating capacitive probe is inserted into a Dynamic Vapour Sorption instrument (DVS-1000, SMS, UK) such that the end-view sensor is positioned below the flat stainless steel microbalance pan (Fig. 1). The same configuration on a bench-top setup has been previously used by Kwek et al. ${ }^{6}$ The DVS cell surrounding the sensor is made of brass in order to provide shielding from external electric fields. The brass cell and hanging wire are grounded, whereas the sample holder is isolated via a polyvinylidene fluoride (PVDF) hook. The data is collected with a rate of 60 points/min using a data acquisition (DAQ) unit multimeter with USB interface (DVM1200, Velleman, Belgium) and recorded via PC-Link software. Environmental conditions such as temperature and relative humidity are kept constant throughout the duration of the measurements by enclosing the system in the incubator and providing a constant dry / wet air mixture, with a total flow rate of $100 \mathrm{sccm}$ through the DVS brass cell.

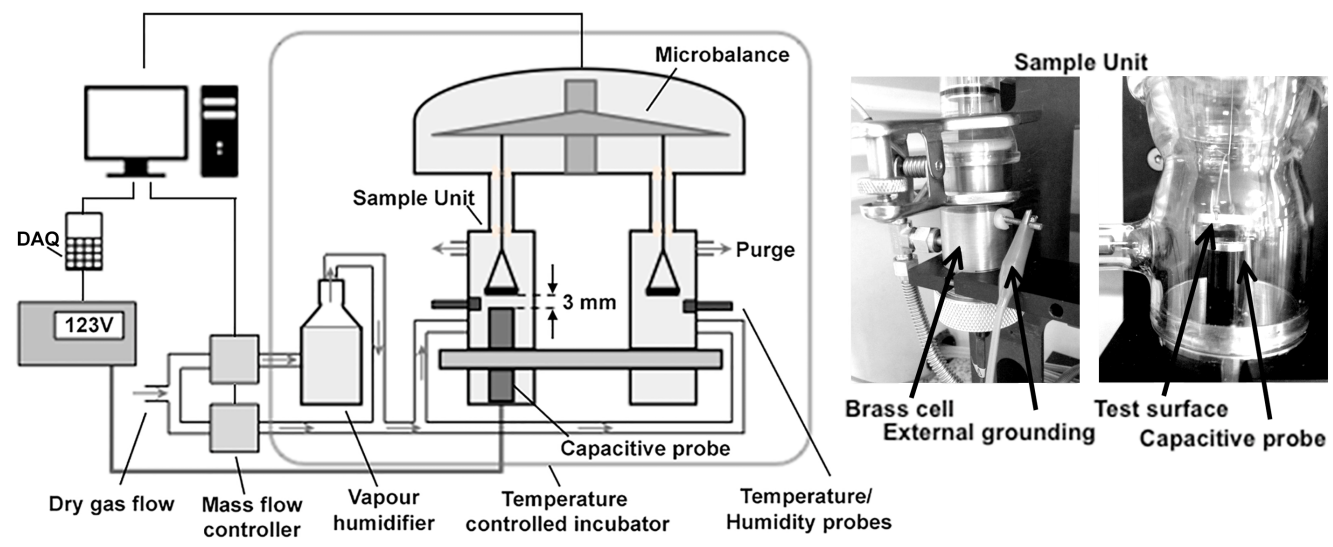

Fig. 1. A schematic diagram of the experimental setup with coupled DVS and vibrating capacitive probe. 
The signal output was confirmed by applying a voltage of $50 \mathrm{~V}$ supplied by a DC power generator (Model PSM3/5A 3CH, BST, UK) to the test surface and then measuring the output with the probe connected to the electrostatic voltmeter. Similarly, an optimum separation distance between the sensor and test surface was determined by measuring the signal output at different positions of probe from the surface under known potential. The $50 \mathrm{~V}$ voltage was applied and the output was measured at $3 \mathrm{~mm}, 8 \mathrm{~mm}, 10 \mathrm{~mm}, 14 \mathrm{~mm}$ and $17 \mathrm{~mm}$ away from the test surface.

Due to the geometry of the DVS, loading of the sample involves displacing the sensor from its original position. According to Eq. (1), such displacement could introduce a change in potential difference, which will then affect the true measurement. The optimum background potential, at which no signal change is observed, was determined by measuring the signal output during sensor displacement with respect to baseline potential.

All measurements were performed at $25{ }^{\circ} \mathrm{C}$ and $20 \% \mathrm{RH}$ unless otherwise stated. Typically, temperature and humidity fluctuations of $\pm 0.1{ }^{\circ} \mathrm{C}$ and $\pm 0.2 \%$ RH were observed within experimental timeframe.

\subsection{Materials}

Glass beads with a particle size distribution of 150-210 $\mu \mathrm{m}$ (Polysciences Europe GmbH) were used as received to test the setup and method adopted. Samples were stored under controlled 
environmental conditions $\left(25{ }^{\circ} \mathrm{C}, 30-35 \% \mathrm{RH}\right)$ in a grounded conductive container for $24 \mathrm{hr}$ prior to and in between each measurement.

\subsection{Procedure for electrostatic measurements}

Prior to each measurement, the stainless steel measuring pan is washed with deionised water and ethanol, allowed to dry and placed inside the DVS at the desired temperature and humidity. The system is allowed to discharge the charge accumulated on the surface of the pan, after which the pan is grounded via a conductive rod once the DVS cell remains closed to dissipate any residual charges. After 10 minutes, the grounding is removed and the background signal is recorded. Once a satisfactory baseline is obtained, the cell is opened and between 15-20 mg of powder is loaded onto the sample holder using a U-shaped stainless steel spatula. The DVS cell is closed and the initial charge of the sample and its subsequent charge decay are measured.

\section{Results and discussion}

A vibrating capacitive probe has previously been shown by Kwek et al. to be capable of measuring the electrostatic potential of charged powders. ${ }^{6}$ However, preliminary tests with the coupled system revealed that a large number of factors may affect the overall electrostatic field and therefore calibration and optimisation are required. 


\subsection{Calibration and method optimisation}

A DC voltage supply has been used to verify the voltage recorded by the probe. The probe was placed at the recommended distance from the test surface and an external voltage of $50 \mathrm{~V}$ was applied to the surface. The starting potential was set as a step change from $0 \mathrm{~V}$ to $-20 \mathrm{~V}$ and from $-20 \mathrm{~V}$ to $0 \mathrm{~V}$. The output was measured with the capacitive probe as the voltage was varied as shown in Fig. 2. In both cases, the overall change in the potential is equal to $50 \mathrm{~V}$. However, the absolute value measured depends on the starting potential and hence $30 \mathrm{~V}$ is detected when $50 \mathrm{~V}$ is applied at $-20 \mathrm{~V}$ background. Therefore, the potential captured is always relative to the background potential.

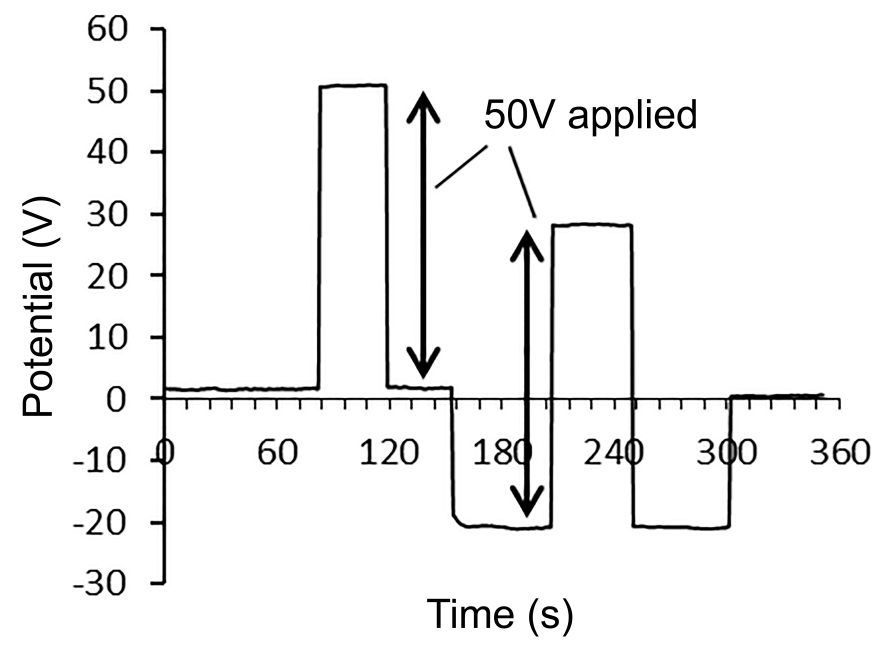

Fig. 2. Output potential verification with respect to applied DC voltage.

From Eq. (1), it is clear that the potential measured by the probe is a function of the separation distance between the probe and the test surface. In order to determine the optimum position of the probe from the sample holder, a potential of $50 \mathrm{~V}$ was applied to the surface and the output measured as a function of distance from the surface. 
Fig. 3 shows that as the separation distance increases, the potential measured drops significantly. This signal drop is more pronounced compared to results found by Kwek et al. as a high resolution, more sensitive probe is used for this method. ${ }^{6}$ The optimum separation between the sensor and the test surface is $3 \mathrm{~mm}( \pm 0.5 \mathrm{~mm})$ and the probe was set in a fixed position throughout all measurements.

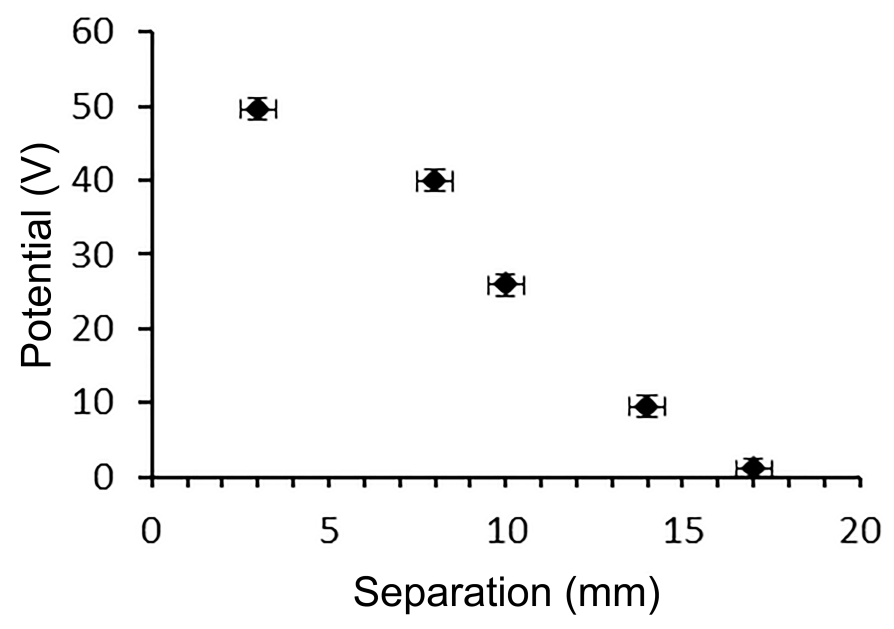

Fig. 3. Output potential signal as a function of distance from the test surface.

Furthermore, in order to load a sample, the DVS cell has to be opened which results in the sensor being moved away from the test surface. As the cell is subsequently closed, the initial separation is restored. However, unless the true potential of the surface is $0 \mathrm{~V}$, the process of shifting the probe will introduce a change in potential (Eq. (1)). The relationship between the background potential and change in potential upon probe dislocation was determined at $25{ }^{\circ} \mathrm{C}$, with a constant air flow rate of $100 \mathrm{sccm}$ and two humidity levels: $20 \% \mathrm{RH}$ and $50 \% \mathrm{RH}$. The results show that there is a linear correlation between these two variables (Fig. 4(a) and (b)) with no change in potential observed for the background potential with approximate value of $2 \mathrm{~V}$. 

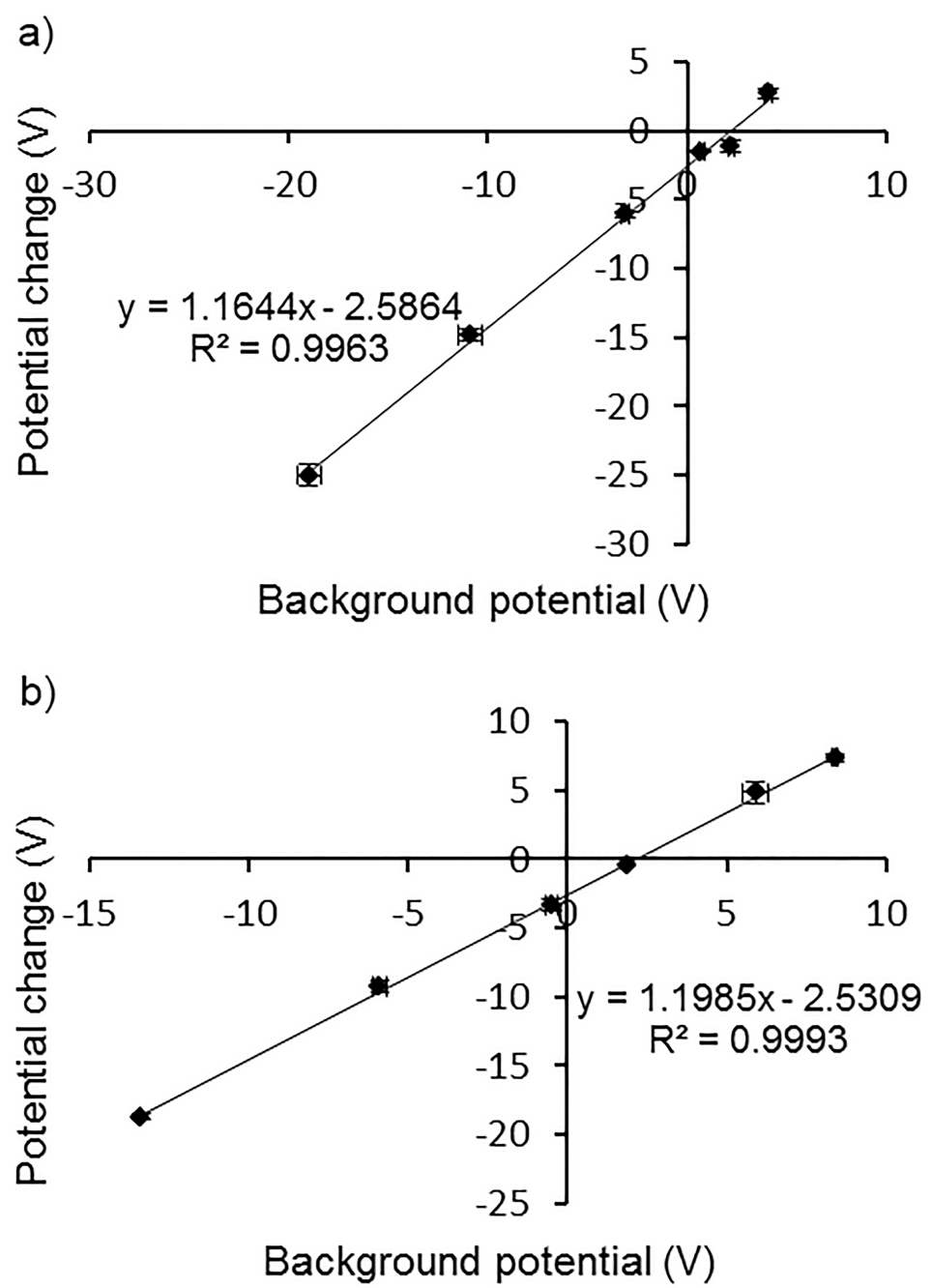

Fig. 4. Potential change as a function of background potential at $25^{\circ} \mathrm{C}$ and a) $20 \% \mathrm{RH}$ and b) $50 \%$ RH.

Based on Eq. (1), it would be expected that no change in potential should be observed for a background potential equal to $0 \mathrm{~V}$, as this would assume the charge of the surface to be zero. This offset by $2 \mathrm{~V}$ may be due to either coupling effect with the shielding cell being at $0 \mathrm{~V}$ potential, distortion of the electric field at large distance from the test surface, the equipment resolution, or a combination of these factors. 


\subsection{Verification of reproducibility}

The major challenge in performing electrostatic measurements is the ability to reproduce experimental results. It is known that the process of charge accumulation and decay is very complex and depends on both material-specific properties, type of interaction causing charging as well as the environmental conditions at which triboelectrification and subsequent discharge

occur. ${ }^{5,9}$ In order to limit the number of factors leading to data variation due to the sample itself, spherical glass beads with a narrow particle size distribution $(150-210 \mu \mathrm{m})$ have been chosen to test the setup. This approach ensures no preferred orientation is adopted by the sample particles and the surface composition is homogenous unlike for most pharmaceutical powders, which could lead to variations in the extent of triboelectrification. Blank experiments under identical conditions are performed to assess the method.

Once the test surface is grounded and allowed to discharge prior the measurement, no additional noise is introduced due to any step involved in the procedure adopted, and hence the background signal is a flat line. Fig. 5 shows an example of the signal recorded when charged glass beads are introduced into the DVS cell. 


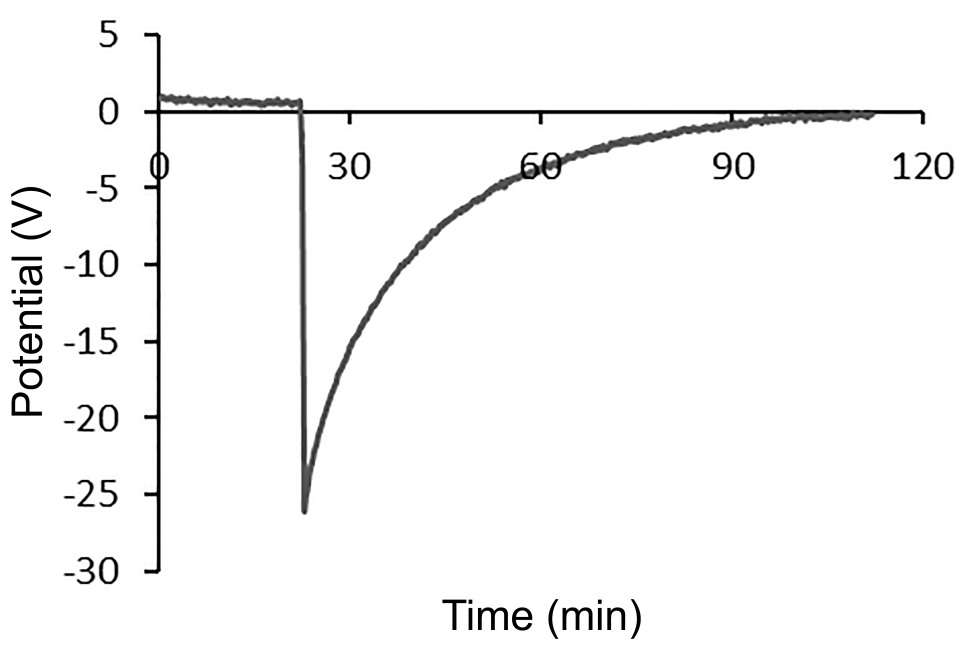

Fig. 5. Potential decay curve recorded for glass beads $(150-210 \mu \mathrm{m})$ negatively charged by stainless steel surface.

Upon contact with a stainless steel surface, glass beads acquire negative charge that is represented by the initial sharp peak. The minimum value recorded corresponds to the maximum potential and therefore charge received by the sample. The signal then becomes less negative with time, returning to its initial value as the charge decays from the sample. The half-life and charge decay rate constant of the sample may be obtained from the curve providing an indication of the extent of the triboelectrification of the beads prior to their introduction to the sample holder

The variation in signal recorded between individual measurements on the same material is shown in Fig. 6. 


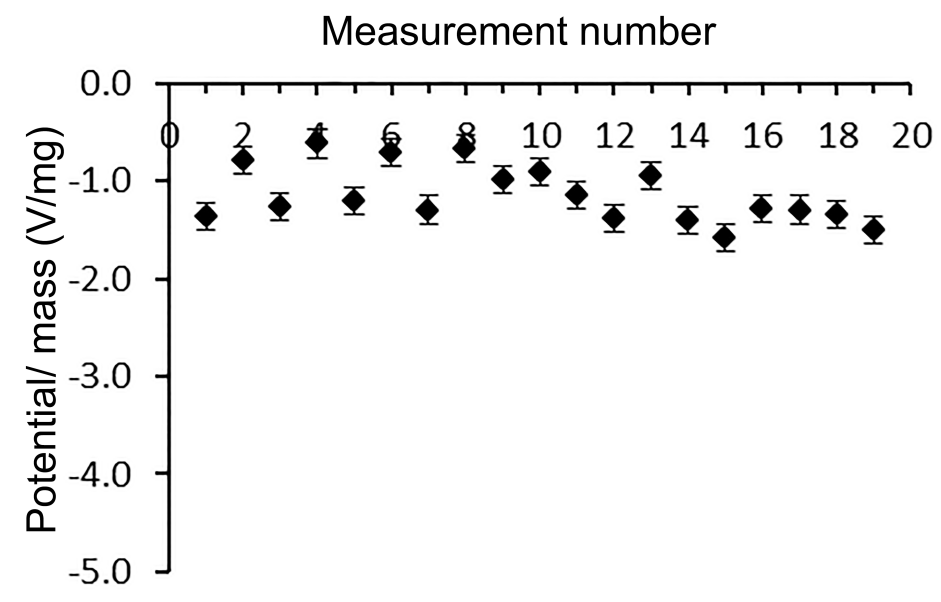

Fig. 6. Reproducibility data on glass beads $(150-210 \mu \mathrm{m})$ negatively charged by stainless steel surface.

Comparing the maximum values recorded, normalised with respect to mass of the sample measured, there is a reasonably good repeatability between the 19 measurements performed. As all measurements are performed for one material only, and no comparison between different surfaces is made, normalisation of signal with respect to mass is applied. Overall, an average value of $-1.2 \pm 0.3 \mathrm{~V} / \mathrm{mg}$ was recorded for the sample. The overall potential measured is relatively low, which is expected for these rather large and smooth particles.

The coefficient of variation of $25 \%$ for 19 measurements presented in Fig. 6 is comparable to values obtained previously by Kwek et al. ${ }^{6}$ which ranged from $4.7 \%$ to $32.7 \%$ using a similar approach. For other techniques values in the range of $40-50 \%$ up to $100 \%$ have been reported. $^{5}$ The measurement-to-measurement deviations are not considered to be due to the experimental setup and procedure. They might result from the differences in the actual interaction of the 
particles with the tribocharging surface, even though deposition of particles was performed as consistently as possible. If necessary, manual loading of the sample could be replaced with an automated method, reducing the variability in the extent of triboelectrification between the glass beads and stainless steel surface, thus increasing the repeatability and reliability of the results recorded.

\section{Conclusion}

The results show that the proposed method involving the capacitive probe may be successfully used for measuring the electrostatic potential of powders. By incorporating the sensor into a DVS, the environmental conditions, including relative humidity, can be precisely controlled. The presence of the microbalance allows for measurement of the mass of the powder as well as tracking any changes in mass as a function of time which may occur as a result of moisture uptake, which in turn could affect the charge decay characteristics of the sample. The results also show that optimisation and calibration of the experimental setup allow the incorporation of noncontact charge measurement method into an existing system which could potentially be extended further into in-situ monitoring of the triboelectrification of powders during processing.

\section{Acknowledgements}

The PhD studentship, supported by the Engineering and Physical Science Research Council and Pfizer for Karolina Biegaj, is gratefully acknowledged. The authors acknowledge Dr Daryl Williams for fruitful discussions. 


\section{References}

(1) Bailey, A. G., Electrostatic phenomena during powder handling. Powder Technol. 1984, 37, 71-85.

(2) Matsusaka, S.; Maruyama, H.; Matsuyama, T.; Ghadiri, M., Triboelectric charging of powders: A review. Chem. Eng. Sci. 2010, 65 (22), 5781-5807.

(3) Karner, S.; Urbanetz, N. A., The impact of electrostatic charge in pharmaceutical powders with specific focus on inhalation-powders. J. Aerosol Sci 2011, 42 (6), 428-445.

(4) Wong, J.; Chan, H. K.; Kwok, P. C. L., Electrost in pharmaceutical aerosols for inhalation. Ther. Deliv. 2013, 4 (8), 981-1002.

(5) Ramirez-Dorronsoro, J.; Jacko, R. B.; Kildsig, D. O., Chargeability measurements of selected pharmaceutical dry powders to assess their electrostatic charge control capabilities. AAPS PharmSciTech 2006, 7 (4), E1-E8.

(6) Kwek, J. W.; Jeyabalasingam, M.; Ng, W. K.; Heng, J. Y. Y.; Tan, R. B. H., Comparative Study of the Triboelectric Charging Behavior of Powders Using a Nonintrusive Approach. Ind. Eng. Chem. Res. 2012, 51 (50), 16488-16494.

(7) Bennett, F. S.; Carter, P. A.; Rowley, G.; Dandiker, Y., Modification of Electrostatic Charge on Inhaled Carrier Lactose Particles by Addition of Fine Particles. Drug Dev. Ind. Pharm. 1999, 25 (1), 99-103.

(8) Forward, K.; Lacks, D.; Sankaran, R., Charge Segregation Depends on Particle Size in Triboelectrically Charged Granular Materials. Phys. Rev. Lett. 2009, 102 (2).

(9) Šupuk, E.; Zarrebini, A.; Reddy, J. P.; Hughes, H.; Leane, M. M.; Tobyn, M. J.; Timmins, P.; Ghadiri, M., Tribo-electrification of active pharmaceutical ingredients and excipients. Powder Technol. 2012, 217, 427-434.

(10) Elajnaf, A.; Carter, P.; Rowley, G., Electrostatic characterisation of inhaled powders: effect of contact surface and relative humidity. Europ. J. Pharm. Sci. 2006, 29 (5), 375-84.

(11) Elajnaf, A.; Carter, P.; Rowley, G., The effect of relative humidity on electrostatic charge decay of drugs and excipient used in dry powder inhaler formulation. Drug Dev. Ind. Pharm.

2007, 33 (9), 967-74.

(12) Ireland, P. M., Triboelectrification of particulate flows on surfaces: Part I — Experiments. Powder Technol. 2010, 198 (2), 189-198.

(13) Bailey, A. G., Charging of solids and powders. J. Electrostat. 1993, 30, 167-180.

(14) Kwok, P. C.; Chan, H. K., Electrostatics of pharmaceutical inhalation aerosols. J. Pharm. Pharmacol. 2009, 61 (12), 1587-99.

(15) Peart, J., Powder electrostatics: Theory, techniques and applications. KONA Powder Part. J. 2001, 19, 34-45.

(16) Chow, K. T.; Zhu, K.; Tan, R. B.; Heng, P. W., Investigation of electrostatic behavior of a lactose carrier for dry powder inhalers. Pharm. Res. 2008, 25 (12), 2822-34.

(17) Carter, P. A.; Rowley, G.; Fletcher, E. J.; Stylianopoulos, V., Measurement of Electrostatic Charge Decay in Pharmaceutical Powders and Polymer Materials Used in Dry Powder Inhaler Devices. Drug Dev. Ind. Pharm. 1998, 24 (11), 1083-1088.

(18) Dębska, M., Surface potential decay on triglycine sulfate crystal. J. Electrostat. 2005, 63 (11), 1017-1023.

(19) Kumar, A.; Nath, R., Study of the surface potential decay characteristics in cellulose acetate films. J. Electrostat. 1983, 14, 201-208. 
(20) Othman, N. A.; Piah, M. A. M.; Adzis, Z.; Ahmad, H., Measurement of surface charge distribution on glass insulator using steel mesh. In IEEE International Conference Power \& Energy, 2014; pp 105-108.

(21) Pritchard, D., Electrostatic Voltmeter and Fieldmeter Measurements on GMR Recording Heads. In Electrical Overstress/Electrostatic Discharge Symp. Proc., Anaheim, CA, 2000; pp 499-504.

(22) Noras, M. A., Non-contact surface charge/voltage measurements: Capacitive probe principle of operation. TREK, 2002; pp 1-8. 
For Table of Contents Use Only

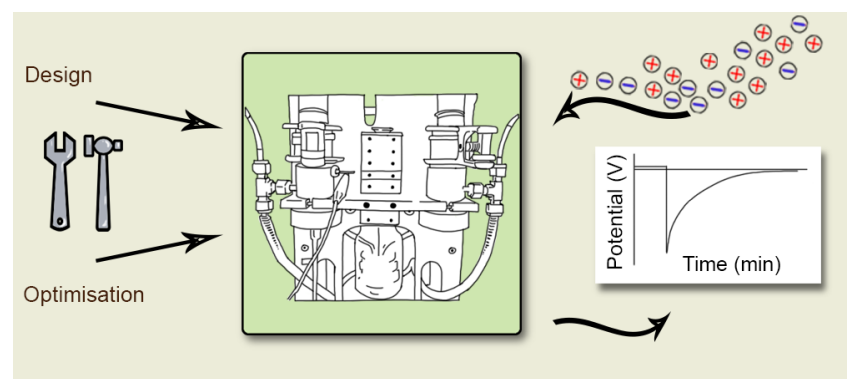

